This item was submitted to Loughborough's Research Repository by the author.

Items in Figshare are protected by copyright, with all rights reserved, unless otherwise indicated.

\title{
Development of a synthetic human thigh impact surrogate for sports personal protective equipment testing
}

\section{PLEASE CITE THE PUBLISHED VERSION}

http://dx.doi.org/10.1177/1754337115582294

\section{PUBLISHER}

(C) IMechE. Published by Sage

\section{VERSION}

AM (Accepted Manuscript)

\section{PUBLISHER STATEMENT}

This work is made available according to the conditions of the Creative Commons Attribution-NonCommercialNoDerivatives 4.0 International (CC BY-NC-ND 4.0) licence. Full details of this licence are available at: https://creativecommons.org/licenses/by-nc-nd/4.0/

\section{LICENCE}

CC BY-NC-ND 4.0

\section{REPOSITORY RECORD}

Payne, Thomas, Sean R. Mitchell, Ben J. Halkon, Richard J. Bibb, and Mark Waters. 2019. "Development of a Synthetic Human Thigh Impact Surrogate for Sports Personal Protective Equipment Testing". figshare. https://hdl.handle.net/2134/18874. 


\title{
Development of a synthetic human thigh impact surrogate for sports personal protective equipment testing
}

\section{Thomas Payne ${ }^{1}$; Séan Mitchell ${ }^{1}$; Ben Halkon ${ }^{1}$; Richard Bibb ${ }^{2}$; Mark Waters ${ }^{3}$}

\begin{abstract}
Synthetic impact surrogates are widely used in the sporting goods industry in the evaluation of personal protective equipment (PPE). Existing surrogates, exemplified by those used in safety standards, have many shortcomings, primarily relating to their mass, stiffness, geometries and levels of constraint which limit their biofidelity and subsequent usefulness in PPE evaluations. In sports, absence from competition is a primary severity measure for injuries; consequently blunt trauma injuries, such as contusions and lacerations become pertinent and serious concerns. It is important, therefore, that synthetic surrogates provide an adequate description of these soft tissues to effectively evaluate injury risk. A novel, multi-material human thigh surrogate has been presented with consideration to the tissue structures, geometries and simulant materials used. This study presents the detailed development stages undertaken to fabricate a multi-material synthetic soft tissue surrogate with skin, subcutaneous adipose and muscle tissue components. The resultant surrogate demonstrates the successful use sequential moulding techniques to construct a full-scale anatomical human impact surrogate which can be used in PPE testing.
\end{abstract}

\section{Keywords}

sports personal protective equipment, surrogate, silicone, polydimethysiloxane, impact

\section{Introduction}

In the field of sports injury biomechanics, the primary goal is concerned with replicating "real life" injurious scenarios through an understanding of the mechanisms of injury, characterisation of human response to loading conditions and development of protective methods to prevent injury occurrence or limit severity (Viano et al., 1989). Human surrogates attempt to provide an artificial representation of a living human, which can be used to achieve these objectives.

Synthetic human surrogates are commonly used in automotive, defence and medical industries to perform a range of functions ranging from training aids (e.g. epidural injection dummies) (Chase, 1973) to assessing the comfort and fit of personal protective equipment (PPE) (e.g. thermal mannequins) (Crown et al., 1998). However, their most common use is in impact testing domains, to assess the effectiveness of injury prevention measures (Roberts et al., 2007; Hrysomallis, 2009). These measures can vary significantly between contexts dependent on their operational environment and levels of threats. For example, in the automotive industry, loss of life is clearly the principal threat and airbags or other similar vehicle safety systems are often the focus for surrogates; in a military context PPE such as landmine protection boots and armoured vests are often assessed using surrogates to prevent severe debilitation or fatal injury.

\footnotetext{
${ }^{1}$ Sports Technology Institute, Loughborough University, UK; ${ }^{2}$ Design School, Loughborough University, UK;

${ }^{3}$ School of Dentistry, Cardiff University, UK 
The surrogates developed for the automotive and defence industries reflect their function and are engineered to provide an assessment of the fatality risk from their impact scenario. The Hybrid III crash test dummy (Backaitis \& Mertz, 1993), for example, uses rigid body segments based on $50^{\text {th }}$ percentile male anthropometries and assesses injury risk based on feedback from instrumentation (e.g. load cells, strain gauges) rather than real mechanical deformations and stresses.

In the sporting goods industry, however, the threats are far less severe and the seriousness of an injury is often determined by the time a player is absent from competition (Velani et al., 2012). Injuries to athletes performing at a professional level are a significant financial burden to many stakeholders (e.g. sponsors, teams), whilst injuries to recreational players impose a cost in terms of the number of productive societal days lost through injury. Therefore less debilitating soft tissue injuries such as contusions or lacerations become a pertinent concern (Payne et al., 2013).

Human surrogates are commonly used in the sports industry to assess the effectiveness of PPE. Safety standards prescribe the minimum acceptable requirements a garment must satisfy. However, the standards typically use rigid anvils of unrepresentative geometries, constrained to a massive base (e.g. BS:EN 13546:2002 - field hockey goalkeeper clothing; BS:6183-3:2000 - cricket leg protectors). This can lead to inaccurate assessments of PPE effectiveness as many real human response phenomena have been overlooked such as soft tissue compliance and ability to recoil on impact. Specific issues that can be potentially introduced through such tests are differences in:

- The interaction of the PPE with the body segment and subsequent distribution of pressure.

- The magnitude and rate of deformation of the body segment and PPE.

- The magnitudes of stress and strain in the body segment.

- The proportion of strain energy absorbed by the PPE and body segment.

- The magnitude of stress and strain experienced by the PPE and extent of failure.

To assess the pertinent sports soft tissue injuries a better representation of human soft tissues is required. It is hypothesised that more biofidelic impact surrogates can be made to provide a more human-like interface that can offer a more realistic assessment of PPE effectiveness and a better understanding of injury mechanisms.

Previous research (Payne et al., 2015a, 2015b) has demonstrated, using computational modelling techniques, the potential for layers of distinct synthetic human tissue simulants to produce a more detailed biofidelic surrogate response. This study aims to disprove the null hypothesis that these materials cannot be used to construct a geometrically accurate synthetic human thigh surrogate by proving that:

1. More biofidelic multiple soft tissue synthetic surrogates can be designed incorporating appropriate anatomical simplifications to avoid excessive cost.

2. Manufacturing challenges can be overcome and a full-scale thigh surrogate can be fabricated using newly developed synthetic human soft tissue simulants by employing modern manufacturing processes. 


\section{Surrogate constituents and moulding approaches}

\section{Surrogate component selection and definition}

For the purposes of sports PPE research and development, three elements are considered integral to the development of a synthetic soft tissue surrogate: the range of tissue structures modelled, shape, and simulant materials used.

To develop a viable surrogate, consideration must be afforded to the selection of appropriate levels of anatomical simplification. It is important that the structural complexity of the synthetic surrogate is advanced incrementally, rather than attempting to create a complicated multi-body system prematurely, to avoid generating complex systems that rely on unverified assumptions (e.g. interactions between tissue structures, degrees of freedom at tissue interfaces) that incur additional cost without establishing a proportionate benefit.

In the human body, there are many complex interacting tissue structures which contribute to its mechanical impact response. To date, most impact surrogates, particularly those used in the sporting goods industry, have typically only considered a single soft tissue simulant to replicate the composite response of all tissues in the body segment (Ankrah \& Mills, 2003; Hrysomallis, 2009). This approach overlooks many important human response phenomena such as variable tissue stiffness, relative movement between structures, pressure distribution and deformation of tissues distant from the impact site. Recent research (Payne et al., 2015a, 2015b) has indicated that a structured multi-material surrogate can provide a more "biofidelic" representation of human impact response than single material constructions.

In the human thigh region (Figure 1(a)), the femur is established as an integral component to provide structural support and stiffness to the body region when under impact. Muscle, skin, and subcutaneous adipose are considered to be the principle soft tissue structures contributing significantly to the mechanical impact response in the body region. Muscle tissue constitutes the bulk of soft tissue in the human limbs and accounts for 40-50\% of human body mass (Wang et al., 1997; Salem et al., 2006). Skin is the outermost layer of tissue on the human body and represents a protective barrier from mechanical trauma and stiff interface surrounding other tissues (Edwards \& Marks, 1995; PaillerMattei et al., 2008). With the exception of muscle and skeletal tissues, skin represents the largest organ, constituting approximately 5.5\% of body mass (Goldsmith, 1990). Subcutaneous adipose, also known as the hypodermis, is a connective fatty tissue located between the dermis and the aponeuroses and fascia of muscles, and is bonded strongly to the dermis. Adipose plays an important role as a mechanical load absorbing and distributing member that absorbs shock and protects against local stresses (Robbins et al., 1989; Miller-Young et al., 2002; Geerligs et al., 2008; Sims et al., 2010; Comley \& Fleck, 2012; Alkhouli et al., 2013).

To fabricate a surrogate embodying the full complexity of human substructures as shown in Figure 1(a) (i.e. including muscle compartments, fascia, nerves, vascular structures) would require significant material processing and mould tooling expense without, it is believed, significant additional return in terms of value in sports impact/PPE assessment. To improve sports impact surrogates significantly, but incrementally and economically justifiably, the diversity of tissues structures present were simplified into three distinct types. The resulting simplified representation is shown in Figure 1(b). 


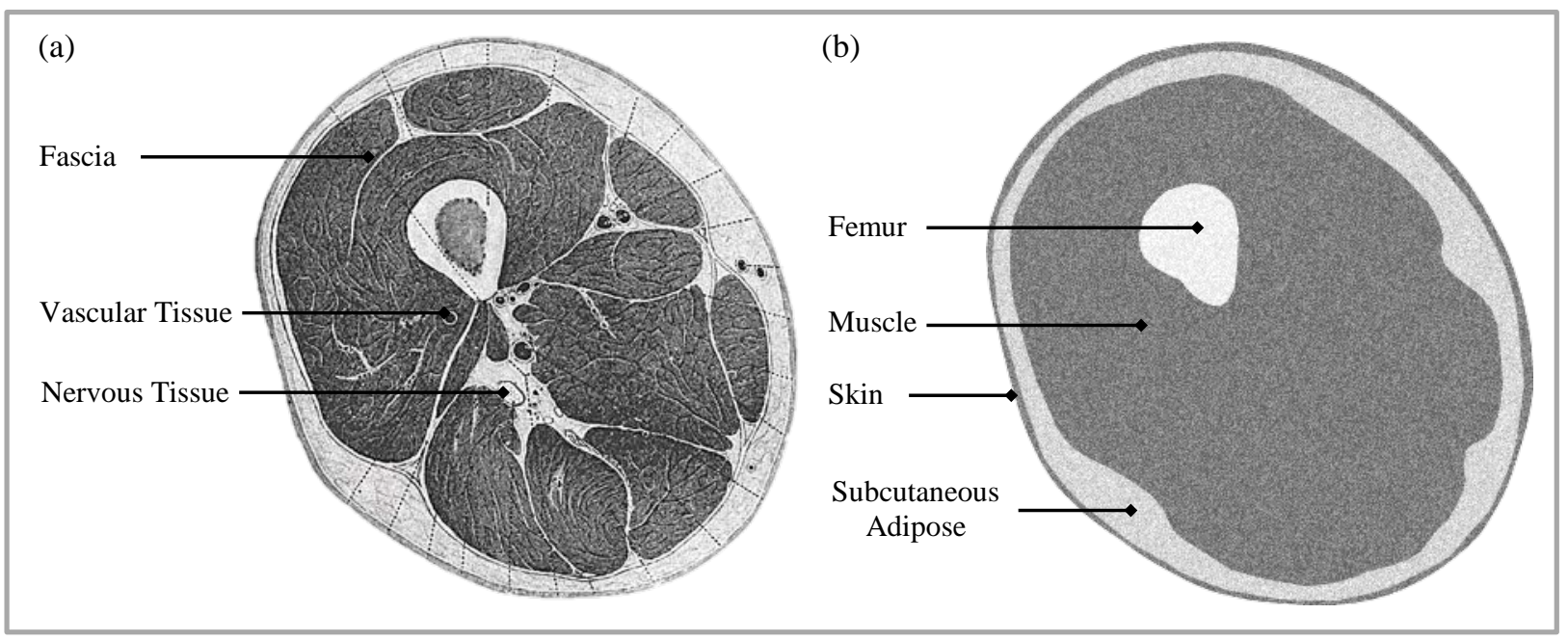

Figure 1. (a) Sagittal plane section through the human thigh showing the diversity of tissue structures (Gray, 2000); (b) Anatomical simplification of the thigh for synthetic surrogate.

The visible human project (VHP) dataset (Ackerman, 1998) was used as a reference dataset to establish the required anthropometric parameters for the surrogate thigh. The VHP in an online database of axial computed tomography (CT) transverse plane scans at $1 \mathrm{~mm}$ intervals from a white, age 38 year old, $50^{\text {th }}$ percentile $(90.2 \mathrm{~kg}, 180 \mathrm{~cm})$ male of US origin taken shortly after death (Spitzer et al. 1996).

To generate the required 3D volumetric geometries, data points from the cross-sectional images were first extracted in image processing software to provide tissue boundaries representative of the three major soft tissue structures. Images were used at regular intervals on the femur, in locations where other skeletal structures (i.e. the pelvis and patella) were not present in the soft tissue. The data points were then converted into a series of splines at each cross-section and reconstructed in computer-aided design (CAD) software to provide a three-dimensional (3D) representation of the soft tissue structure relative to the femur (Figure 2).

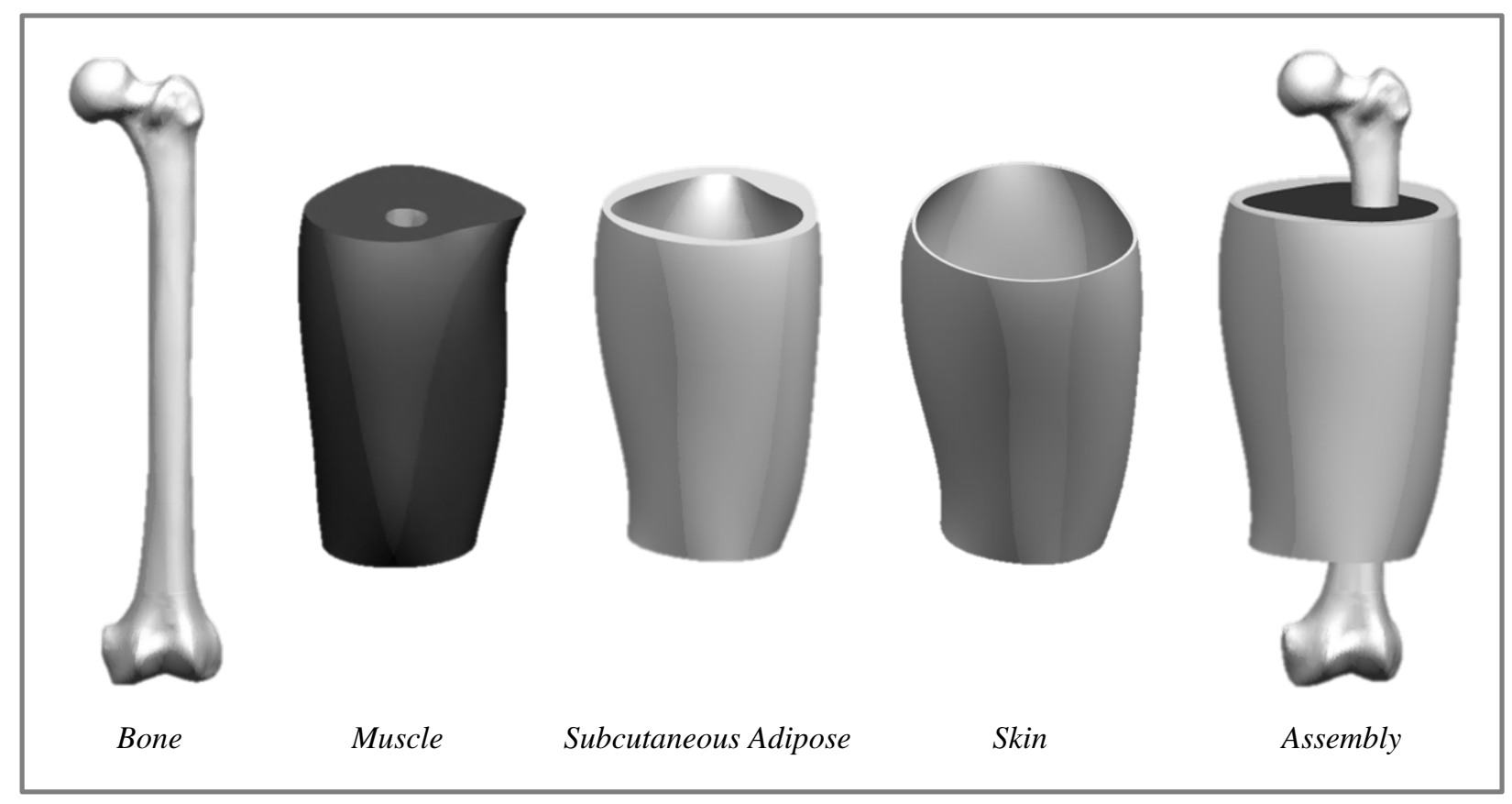

Figure 2. 3D CAD visualisations of the anatomical thigh geometries showing constituent tissue components. 
The materials used in synthetic human surrogates are a key component significantly affecting their behaviour. The simulant materials should ideally match the dynamic stiffness of the human tissue structures to provide as biofidelic an impact response as possible (Saraf et al., 2007). Silicone rubbers have been previously used as a soft tissue simulant (Hrysomallis, 2009; Cronin et al., 2011; Davison et al., 2013) due to their durability, the consistency of their response and their comparable material density (approximately $1000 \mathrm{~kg} \cdot \mathrm{m}^{-3}$ ). In this research, bespoke two-part additive cure polydimethysiloxane (PDMS) silicones were developed to match the specific mechanical properties of each of skin, adipose and muscle tissues. The formulations, fabrication procedures and mechanical testing methods used for each identified simulant are detailed in Payne et al. (2015a, 2015b).

The surrogate has been designed with the capability to incorporate either relaxed or contracted muscle tissue using the alternative formulations established in Payne et al. (2015a). However, in the first instance, relaxed muscle tissue properties were selected for the initial surrogate as a priority since impact injuries are believed to be both more prevalent and serious when the participant is not anticipating the impact (Conforti, 2013). Muggenthaler et al. (2008) also suggest that the human monosynaptic stretch reflexes occur between 30 and 60 ms such that, in the majority of sports impacts, the most severe compressive stage is likely to have occurred before the body actively responds.

A large fourth generation composite Sawbone ${ }^{\mathrm{TM}}$ femur (left limb) was selected as the surrogate skeletal component. The femur is constructed from short glass fibre reinforced epoxy, pressure injection moulded around a rigid polyurethane core (with a hollow intramedullary canal) to represent cortical and trabecular bones respectively. The composite femur bone has been shown to exhibit comparable mechanical responses to human bone under quasi-static loading conditions (Chong et al., 2007; Gardener et al., 2010).

\section{Moulding approaches}

The proposed synthetic thigh surrogate soft tissue components needed to be moulded around the composite femur bone. The key requirements established for superior synthetic surrogate biofidelity were:

- Accurate geometric representation of outer and inner surface morphologies,

- Individual representative cross-sectional thicknesses for each tissue type,

- Continuity within tissue volume, without voids or other moulding flaws (e.g. contamination),

- Adhesion at tissue boundaries, and

- Consistent material properties within tissue boundaries.

Achieving this, with the surrogate elements previously documented, presented a series of challenges:

- Location and alignment of complex surface geometries. At each process stage, the tissue simulant moulds must be aligned in a consistent orientation relative to the skeletal component.

- Mixing consistency. It is important that the mechanical properties of each tissue are continuous throughout despite the significant volumes of each simulant to be moulded.

- Work times of silicones. Each simulant contained different concentrations of cross-linker. Silicones with a greater concentration of cross-linker have shorter work times; the skin simulant, in particular, had a particularly short work time of approx. $120 \mathrm{~min}$. The different volumes of uncured tissue simulant must flow through the available apertures well within material work time. 
- Viscosity of silicones vs. flow aperture variation. The constituent formulations of the simulants affect their viscosity. The adipose simulant, in its uncured state, was the most viscous, while the skin simulant had the lowest viscosity. The challenge presented was related to the size of the cavity for each tissue type relative to the viscosity of the silicones.

- Ease of void inclusion. Owing to the mixing volumes, geometries of the mould cavities and viscosities of silicones, voids and other moulding flaws are easily introduced.

One of the most significant challenges when conceiving the manufacturing process was achieving an accurate inner and outer skin geometry. If the adipose simulant were stiffer, a simple nested three stage moulding process (muscle-adipose-skin) with increasing girth would suffice, but since the adipose layer is the softest and does not retain its shape well such an approach was found to be unviable. Forming the skin layer, before introducing the adipose, using a mould tumbling process was considered but discounted because of the difficulty controlling the skin thickness and cure. The solution adopted was to introduce a sacrificial lost-wax like moulding stage using gelatin to define the inner skin boundary.

\section{Surrogate moulding process}

\section{Process stages}

To retain the structural integrity and mechanical properties of the PDMS silicone simulants they each needed to be moulded in a single attempt. A series of process stages were employed to achieve this; an overview of the resulting multi-stage moulding process is shown in Figure 3, with each phase described as follows:

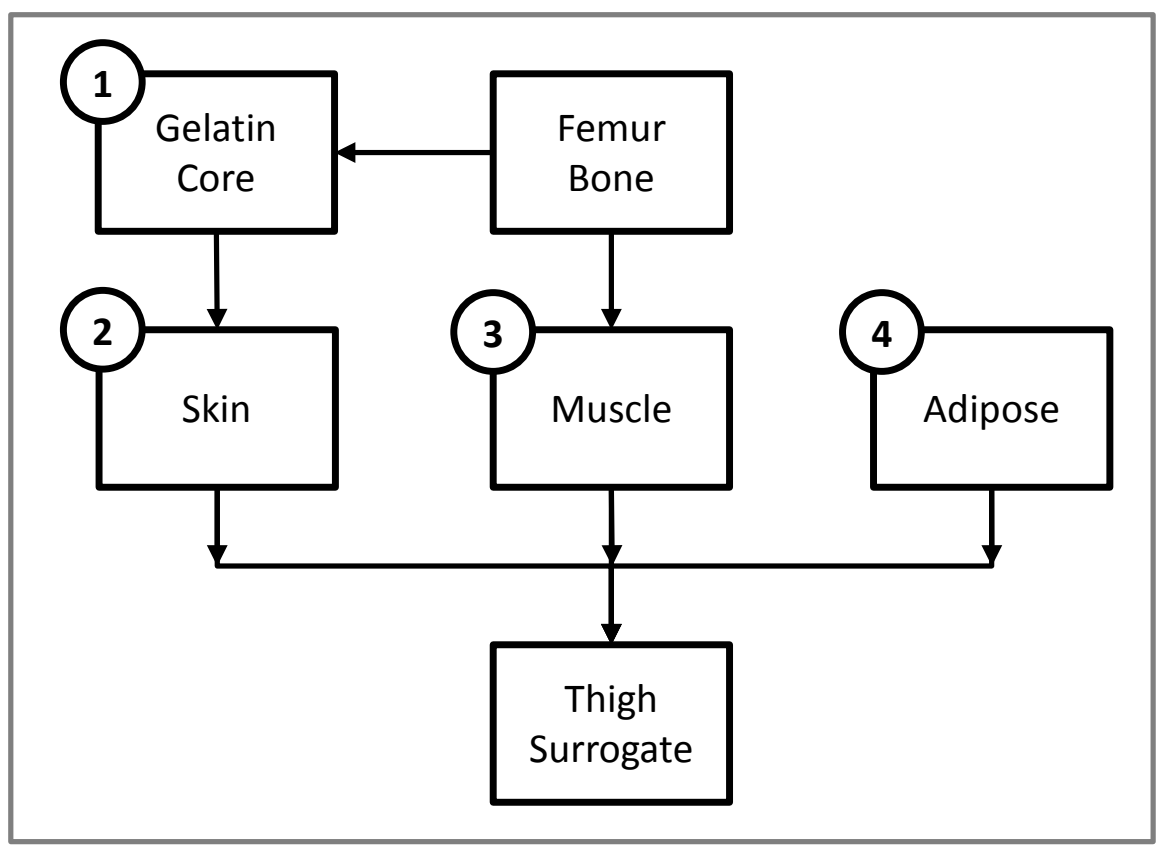

Figure 3. Process stage diagram for thigh surrogate development. 
Preparation. The initial stage of surrogate moulding involved the preparation of a support mould components and support structure, including:

(a) Making moulds for: muscle outer surface; skin inner surface; skin outer surface.

(b) Preparing an adaptive structural support system for the moulds to achieve consistent alignment with the skeletal component.

Stage 1. The first manufacturing stage involved the fabrication of a gelatin core required for the moulding of a thin continuous skin layer, including:

(a) Assembling the skin inner mould for the gelatin core in the mould support assembly.

(b) Moulding of the gelatin core around the femur component.

(c) Setting gelatin and removing from disassembled mould.

Stage 2. The skin layer was the first surrogate tissue to be moulded and succeeded the fabrication of gelatin core, including:

(a) Assembling the skin outer mould in the mould support assembly and align the gelatin core using the femur component.

(b) Pouring the skin simulant into the cavity between the gelatin core and the skin outer mould and heat in an environmental chamber to melt gelatin and leave a continuous skin layer.

(c) Removing the skin layer from the mould support structure whilst attached to the outer skin mould.

(d) Cleaning the skin and femur components from gelatin contaminate.

Stage 3. Moulding of the muscle layer represented the third process stage; it consisted of the following constituent phases:

(a) Assembling the muscle outer mould in the mould support assembly around the femur.

(b) Pouring the muscle simulant in the mould and curing in an environmental chamber.

(c) Disassembling mould and removing the muscle-femur component.

Stage 4. The final moulding phase involved the fabrication of the adipose layer and completion of the soft tissue surrogate. The constituent process stages have been listed:

(a) Positioning the skin outer mould with the skin layer attached in the mould assembly around the muscle femur component.

(b) Pouring the adipose simulant into the cavity between the skin and muscle tissues and curing in an environmental chamber.

(c) Disassembling mould and removing surrogate.

\section{Pilot study: small-scale cylindrical surrogates}

To better understand the practicalities of this moulding process and the efficacy of such an approach, a small-scale pilot study was undertaken. Three cylindrical surrogate moulds were fabricated from acrylonitrile butadiene styrene (ABS) using modelling (FDM) machine. The moulds represented the skin outer, skin inner and muscle a fuse deposition layers and were of diameter 65, 62 and $50 \mathrm{~mm}$ respectively. Each mould consisted of four quadrants with a height of $80 \mathrm{~mm}$ which joined to make the main cavity (Figure 4). A base plate was also fabricated from ABS to complete the volume with a simple positioning stud located on the base to ensure a consistent alignment was maintained between moulding stages. 


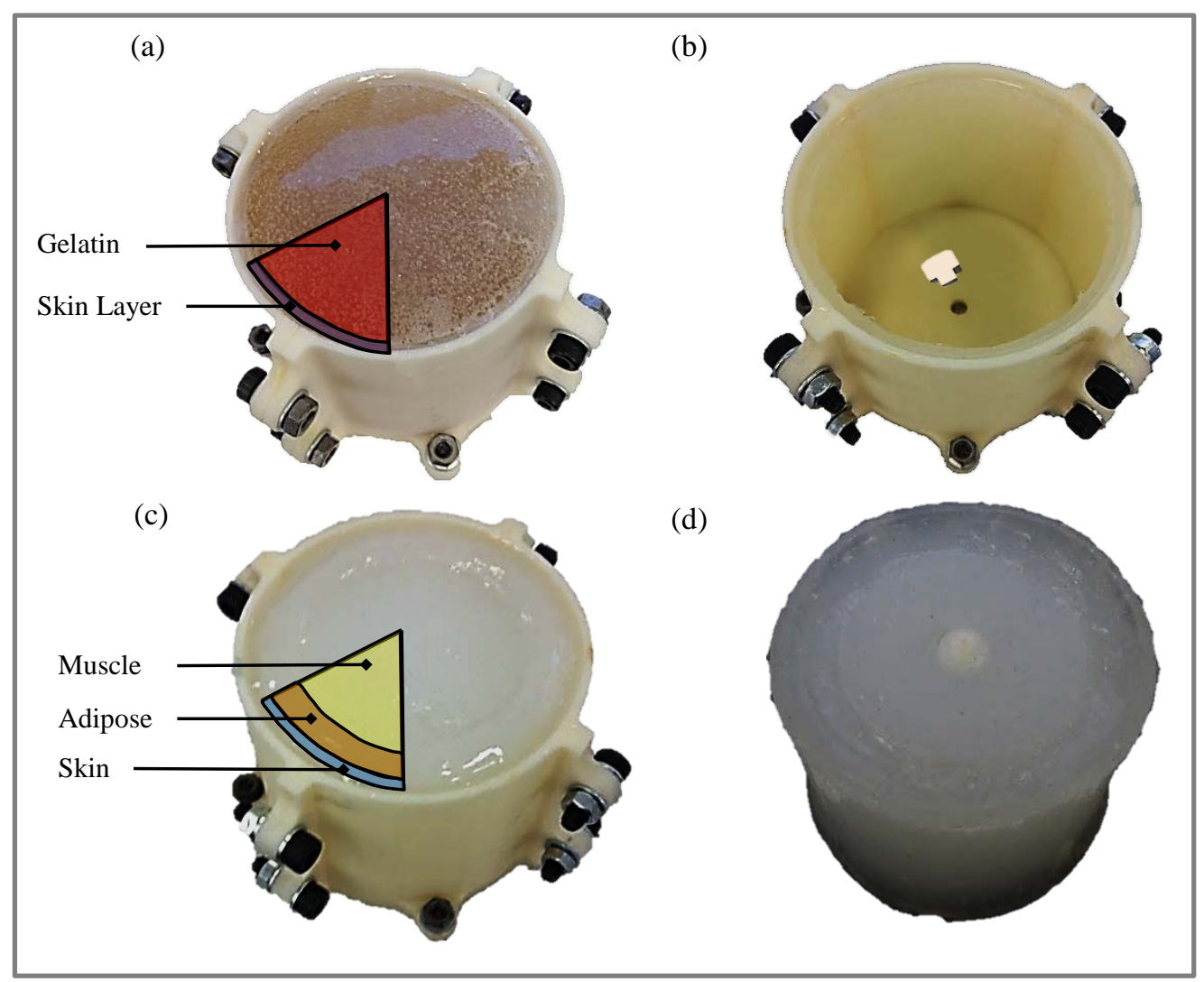

Figure 4. Images of small scale surrogate development stages showing: (a) Stage 1 cured solid PDMS skin simulant and liquid sacrificial gelatin core;(b) Stage 1 cured PDMS skin on outside of mould with positioning stud; (c) Stage 4 final moulding of adipose; (d) Complete surrogate (inverted) showing positioning stud.

At stage 1, the gelatin core was moulded with a stud positioned on the base for subsequent alignment (Figure 4(b)). The PDMS skin material was then poured into the cavity between the ABS mould and the gelatin core; the total pour time was $90 \mathrm{~min}$ for a mould of this scale. Figure 4(a) shows the cured skin simulant in the sacrificial gelatin core immediately after it was removed from the environmental chamber. The liquid gelatin was poured out of the mould and gelatin residue was cleaned from the surfaces with boiling water (Figure 4(b)).

The PDMS muscle simulant was modelled independently, again with a stud located in the base plate. The cured muscle simulant was then positioned back into the skin mould using the locating stud and the adipose was poured in the resultant cavity (Figure 4(c)). The time for the skin simulant to pour into the cavity was a particular issue and took a total of $90 \mathrm{~min}$ for a complete pour.

Given the pouring times observed, scaling up to a full-sized thigh required consideration of more than one pouring point. Exploration of this approach, during the pilot phase revealed difficulties maintaining alignment of the separate soft tissue regions if these were not: (i) adequately established by supporting structures; (ii) aided by even delivery from multiple pour points. Features to enable a rapid, even and consistent flow rate at multiple pour points were subsequently incorporated in the full scale mould tooling designs. 


\section{Full-scale thigh mould tooling development}

Three full-scale anatomical moulds were fabricated out of FDM ABS for the skin outer, skin inner and muscle tissue structures using the detailed moulding approach. The mould assembly for the skin outer mould has been shown in Figure 5 and highlights many of the design features used in each of the cavity moulds.

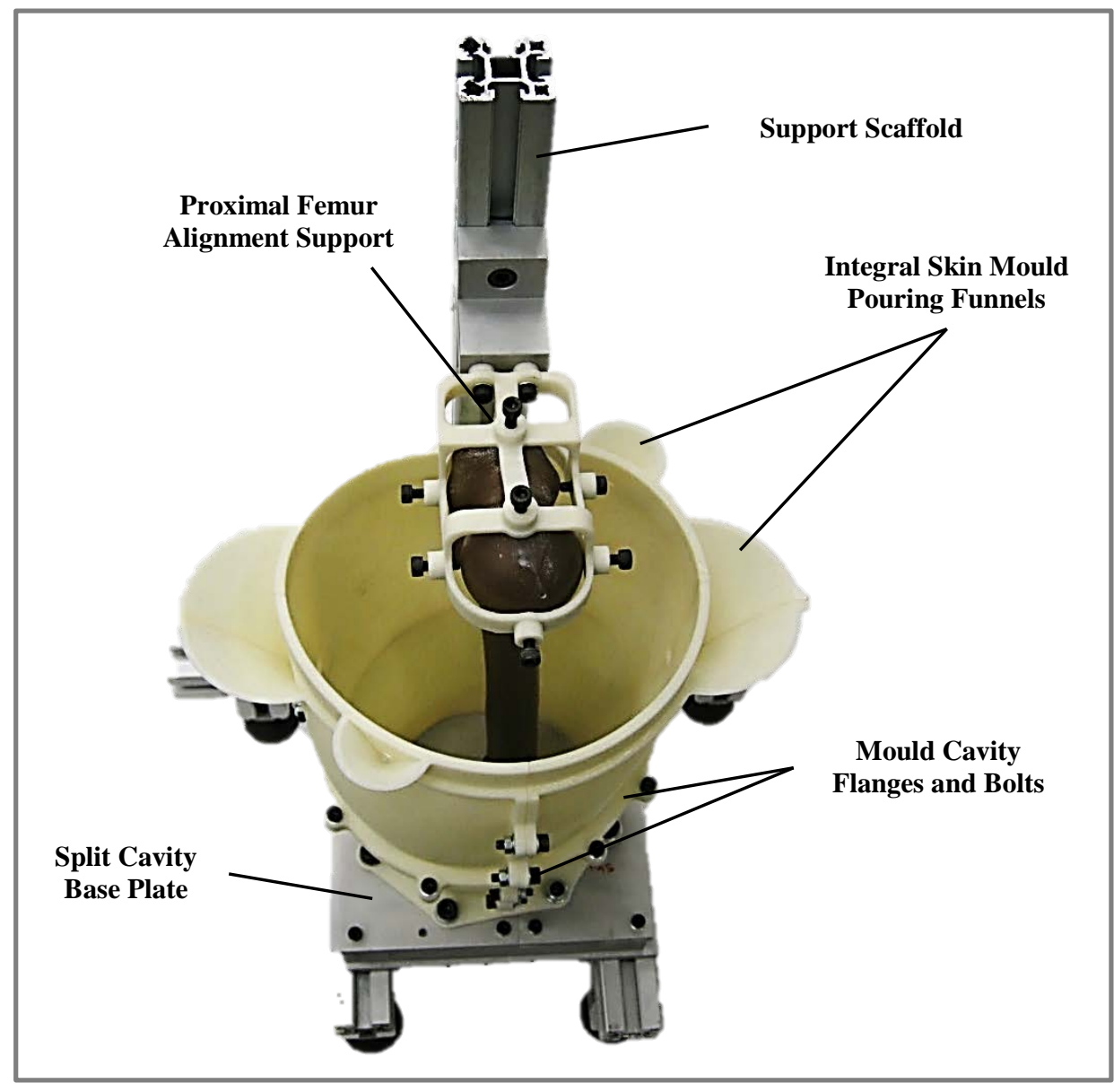

Figure 5. Skin mould assembly with highlighted design features.

Each mould was segmented into eight pieces joined together on flanged surfaces with two $5 \mathrm{~mm}$ bolts to allow economic use of the FDM process (especially use of support material) and easy extraction of the moulded part without damage. The main ABS mould cavities were each mounted on a cavity base plate machined from $10 \mathrm{~mm}$ cast aluminium plate with a clearance cavity around the intersecting femur and a split line through the centroid of the femur on the plate (Figure 6(c)). A scaffold support was constructed using $40 \mathrm{~mm}$ square sections of extruded aluminium profile. The scaffold provided a mounting point to which the mould cavities were attached and a consistent means of alignment for the composite femur. Alignment supports were used at the proximal and distal ends of the femur to rigidly constrain the bone in a specific, nominally vertical orientation. On each support, eight threaded nuts were embedded into the ABS in order that screws could be used clamp and manipulate the composite bone structure for optimal alignment (Figure 6(a)).

The distal femur attachment support was connected to the cavity base plate through an intermediate plate structure, machined from aluminium (Figure 6(b)). The intermediate plate was attached to the scaffold structure at the sides through $5 \mathrm{~mm}$ bolt holes and aligned against the upright scaffold using 
two $5 \mathrm{~mm}$ dowel pins. The intermediate plate and cavity base plate were then connected directly through two $15 \mathrm{~mm} \times 200 \mathrm{~mm}$ aluminium upright supports.

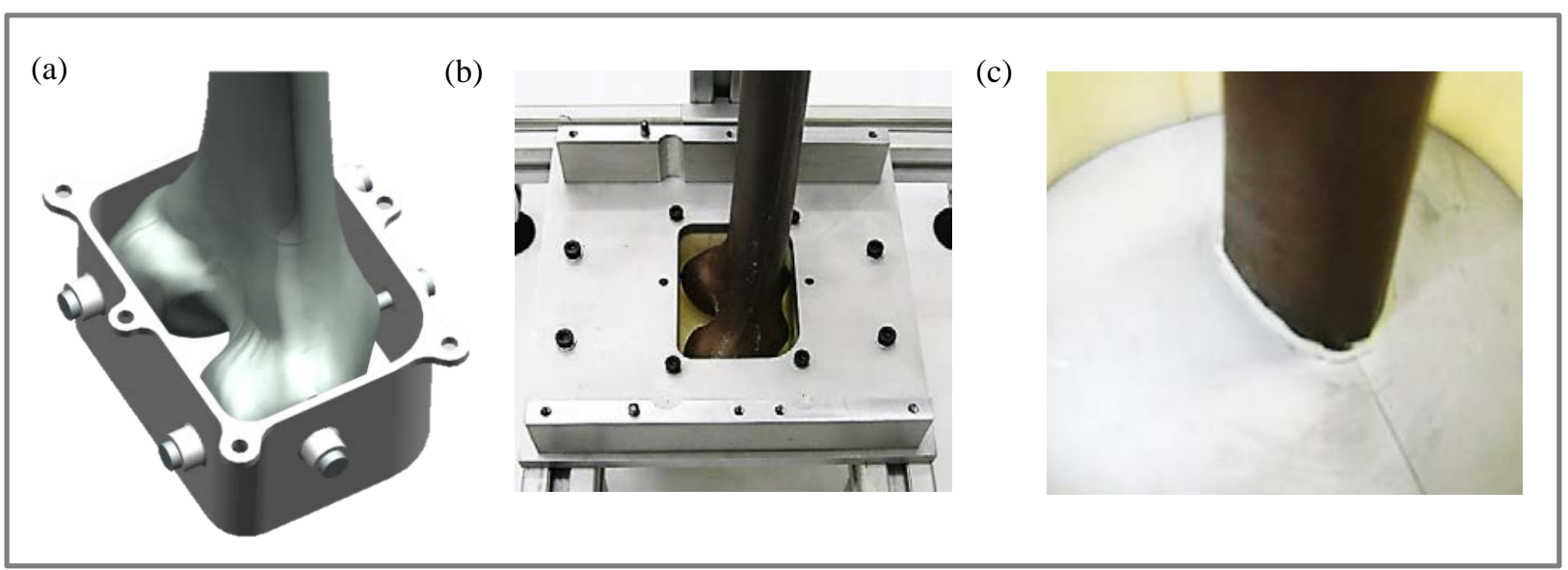

Figure 6. Mould assembly components: (a) CAD image of distal femur attachment; (b) attachments between femur and intermediate base plates (c) cavity base plate femur seal.

As shown in Figure 5, pouring funnels were incorporated into the top of the skin cavity moulds to overcome issues associated with the pour and fill time of the PDMS skin layer. Two primary funnels were positioned on the anterior and posterior sides of the mould, $50 \mathrm{~mm}$ from the top, with $10 \mathrm{~mm}$ diameter feed holes. Two further secondary funnels were positioned above the top of the mould to enable the top $50 \mathrm{~mm}$ of the mould to fill evenly.

\section{Surrogate fabrication}

\section{General procedure}

For each PDMS simulant, the constituents were weighed, mixed and vacuumed following the procedures outlined in Payne et al. (2015a) with 50\% additional material to allow for wastage on the side of the mixing bowl and in the funnels. The quantities of materials used are detailed in Table 1.

Table 1. PDMS simulant constituent quantities $(\mathrm{kg})$ for full surrogate thigh.

\begin{tabular}{lcccccc}
\hline & \multicolumn{3}{c}{ Part A } & \multicolumn{2}{c}{ Part B } \\
\cline { 2 - 7 } Skin & V46 (w/filler) & V31 (w/filler) & V21 (w/filler) & Heat Cure Catalyst & V31 & H301 \\
Muscle & 0.24 & 0.028 & 0.014 & 0.031 & 0.282 & 0.0314 \\
Adipose & 2.23 & 1.03 & 1.03 & 0.48 & 0.857 & 0.0953 \\
\hline
\end{tabular}

Before each phase of moulding the components were assembled in the following order:

1. Bone ends fixed in supports.

2. Proximal femur support attached to intermediate base plate.

3. Dowel pins used to align intermediate base plate to scaffold

4. Intermediate base plate fixed to scaffold.

5. Upright supports attached intermediate base plate to cavity base plate.

6. Cavity segments joined together and attached to cavity base plate.

7. Dowel pins used to align top of cavity to scaffold.

8. Distal femur support fixed to scaffold. 


\section{Skin layer fabrication}

At stage 1, a sacrificial gelatin core was first fabricated (Figure 7(a)). An iterative development study indicated that a $25 \%$ (by mass) gelatin concentration ( 250 Bloom) was optimal to develop a stiff but workable material which would hold its form in a mould. The gelatin was heated at $50^{\circ} \mathrm{C}$ and poured into the mould cavity around the femur component and left to cool for 10 hours at $4^{\circ} \mathrm{C}$ before removal from the mould. At stage 2, the gelatin core was then placed inside the mould assembly with the skin outer cavity assembled around it (Figure 7(b)). The PDMS skin material was then poured into the main mould funnels which were continually refilled until the skin layer reached the pour holes. At this point silicone was poured into the secondary funnels until the entire cavity was filled; the process took a total of 90 min.

The PDMS skin was left at room temperature to partially cure for 120 min before placing the entire mould assembly into the environmental chamber at $90^{\circ} \mathrm{C}$. The mould assembly was removed from the environmental chamber after $180 \mathrm{~min}$ and the molten gelatin was poured out of the mould. Water, just below boiling point, was then brushed onto the inside of the skin layer to remove the gelatin residue before being allowed to cool. The skin layer, attached to the mould cavity, was then carefully removed from the mould support assembly (Figure 7(c)).

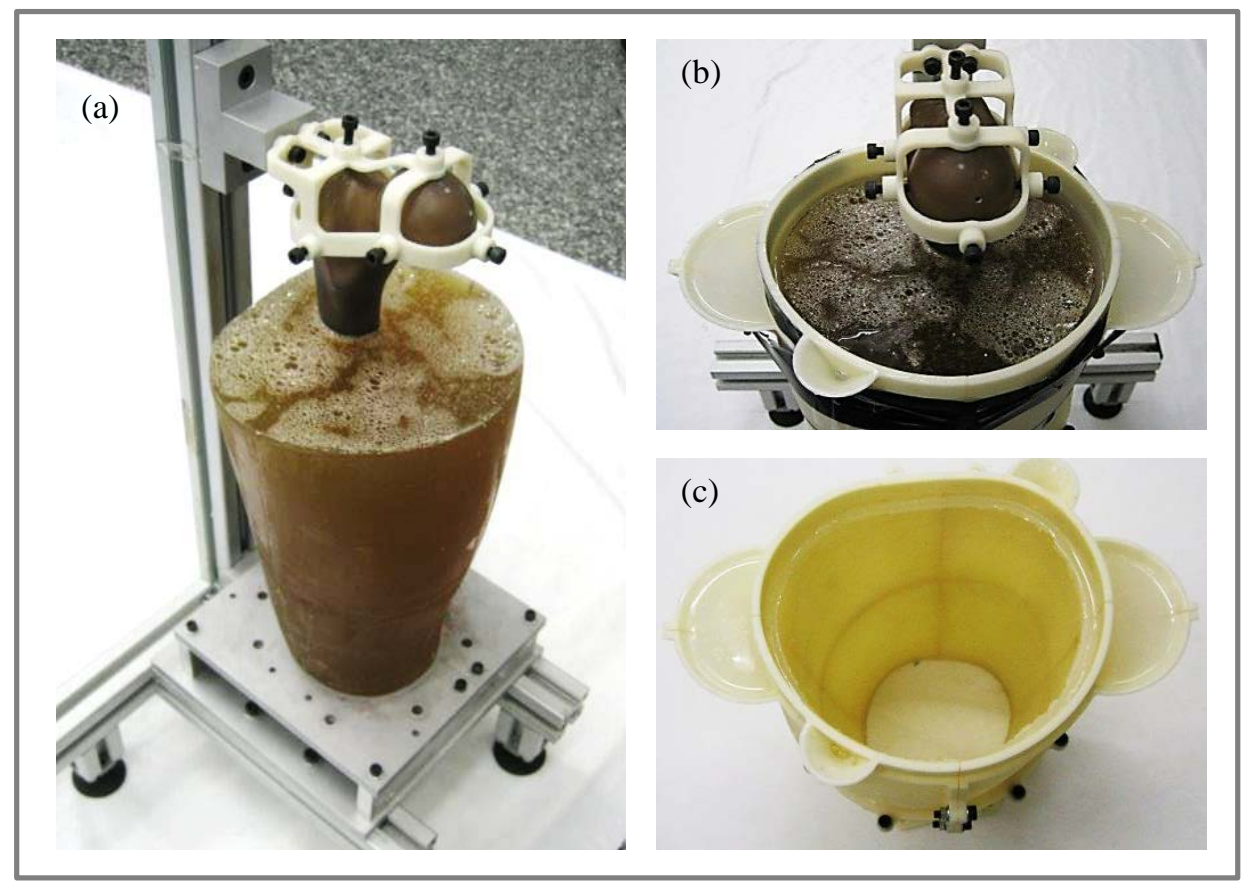

Figure 7. Images showing the stage 1 and 2 development stages for the surrogate skin layer.

\section{Muscle layer fabrication}

At stage 3, the muscle layer was moulded independently to the skin layer with the cleaned composite femur component positioned into the ABS muscle mould cavity. The PDMS muscle material was then poured into the cavity before the mould assembly was placed into the environmental chamber at $60^{\circ} \mathrm{C}$ for 6 hours until fully cured. The muscle layer, attached to the femur, was then carefully removed from the mould assembly (Figure 8). 


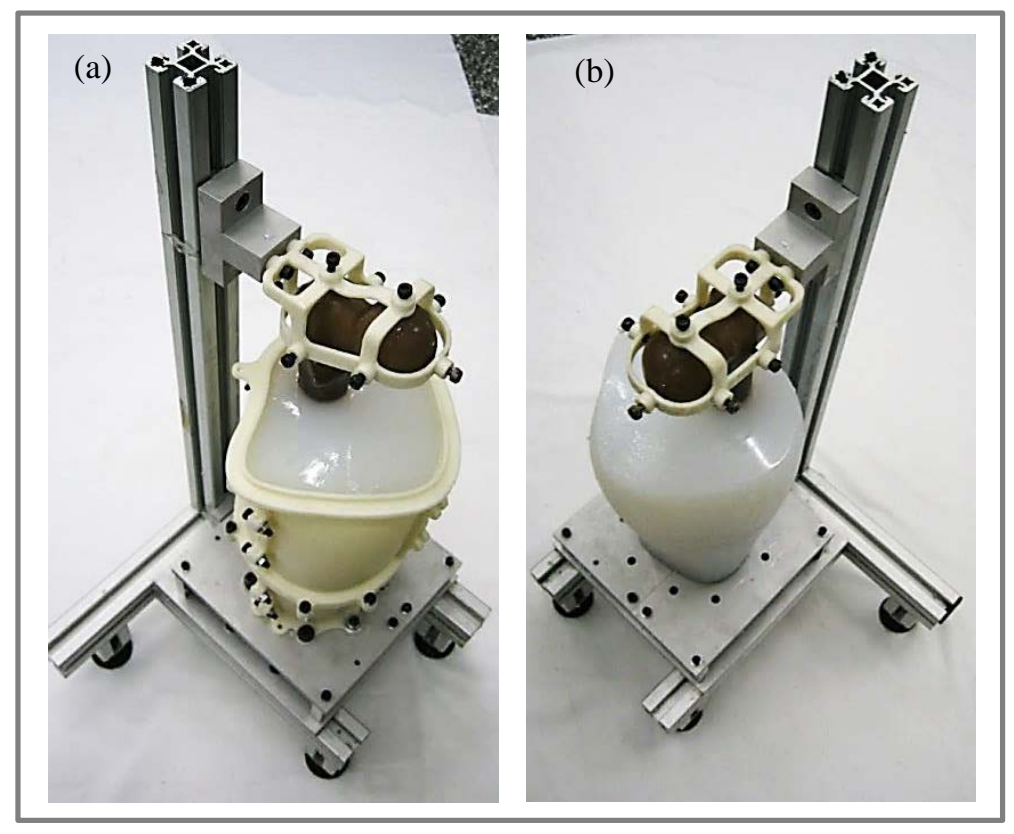

Figure 8. PDMS muscle tissue simulant moulded around femur.

\section{Adipose layer fabrication}

At stage 4, the muscle-femur component was positioned inside the skin mould (with the skin layer attached) and fixed to the mould assembly (Figure 9(a)). The PDMS adipose material was then poured into the resultant cavity between the PDMS skin and muscle material using a positioned funnel (Figure 9(b)). Once filled, the complete mould assembly was placed in the environmental chamber at $60^{\circ} \mathrm{C}$ for 10 hours (Figure 9(c)).

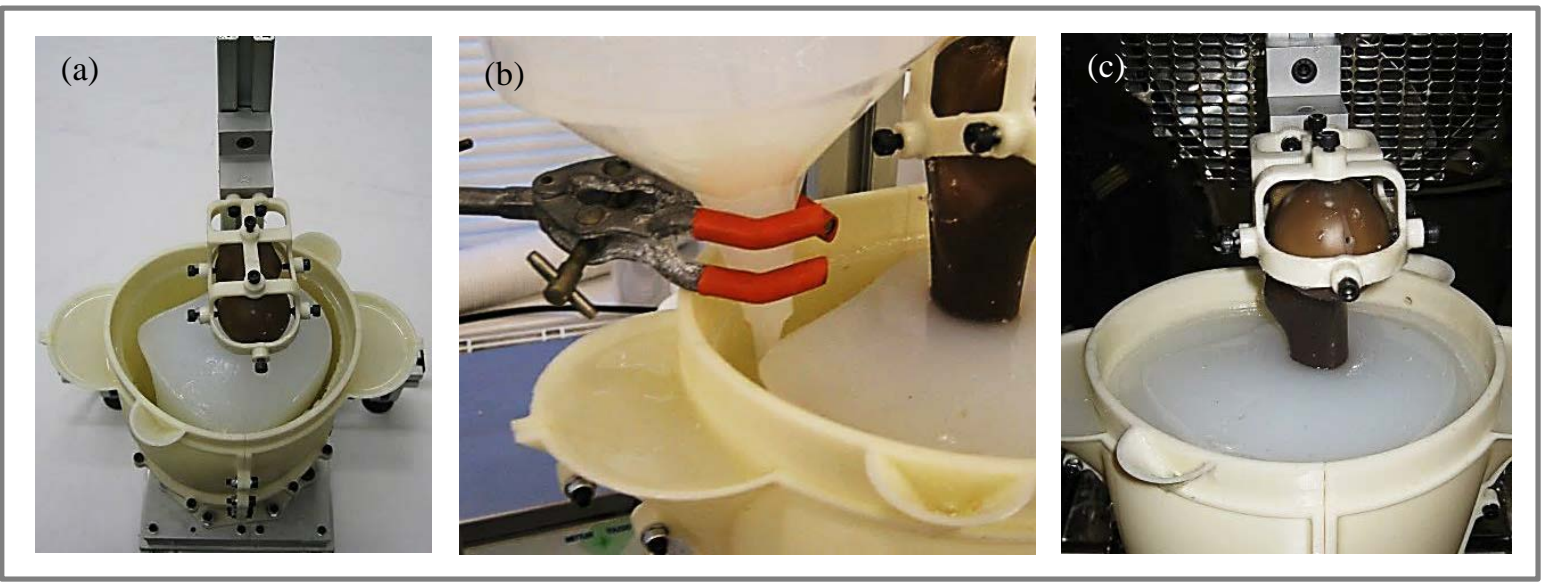

Figure 9. Adipose layer moulding process: (a) muscle positioned in skin mould; (b) funnel used to pour adipose into cavity; (c) full leg assembly in environmental chamber. 
The complete thigh surrogate was allowed to cool for 10 hours and removed from the mould (Figure $10)$.

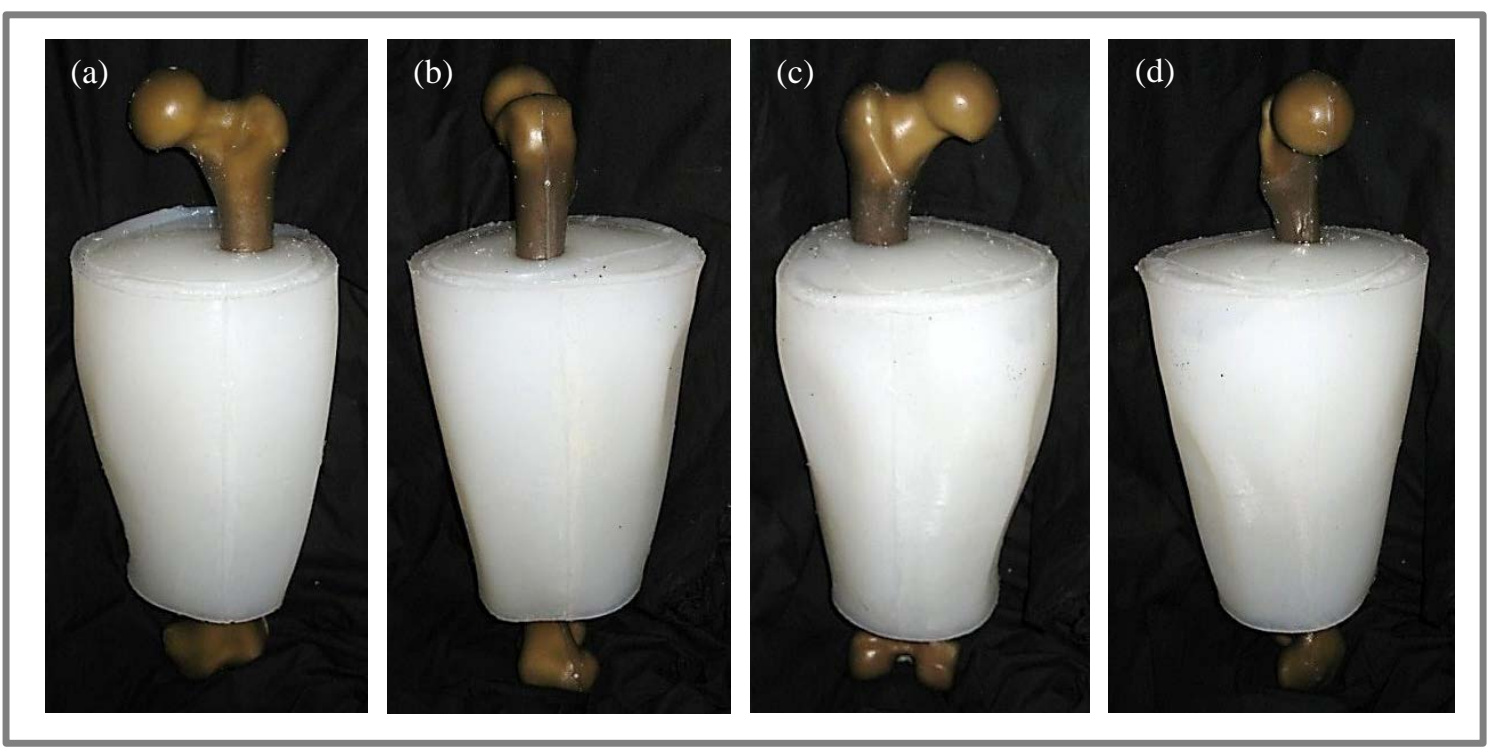

Figure 10. Full synthetic thigh model in: (a) anterior; (b) lateral; (c) posterior; (d) medial views.

The synthetic thigh fabricated provided a full-scale multi-material human surrogate and good surface detail on anterior and lateral surfaces. The posterior and medial regions with large amounts of adipose simulant, however, failed to fully represent the desired geometry and wrinkled (Figure 10(c), 10(d)).

\section{Costing}

A full cost breakdown for the developed surrogate is presented in Table 2.

Table 2. Synthetic thigh surrogate cost breakdown.

\begin{tabular}{ll}
\hline & Cost \\
\hline Silicone & $£ 354$ \\
Gelatin & $£ 7$ \\
Femur & $£ 115$ \\
FDM Mould Components & $£ 1667$ \\
Assembly Support Structure & $£ 48$ \\
Labour \& Machining & $£ 250$ \\
Total & $£ 2441$ \\
\hline
\end{tabular}

\section{Discussion}

\section{Quality of synthetic surrogate}

The overall research aims for this study were principally concerned with determining the feasibility of designing and manufacturing a multi-material soft tissue surrogate for sports impact applications. The resulting surrogate is intended to exhibit dynamic response characteristics that, more closely than has been presented to date, match those of the human equivalent. Anatomical simplifications were initially established through their function in impact applications, resulting in the selection of a four tissue surrogate with: skin, adipose, muscle and bone structures. Geometrical representations of each of these structures were determined through the sampling of anatomical imaging data in such a 
manner as to enable a greater ease of manufacture. The simulant materials used were then selected based on previously determined suitability in sports impact surrogate applications.

The mould assembly was constructed with such features to ensure that the skeletal components and moulds were oriented in a consistent alignment. A combination of precision engineered support structures, dowel pins and femur supports contributed to this. The multi-stage moulding process used enabled each of the tissues to be moulded individually and sequentially in a 'single shot' mould. This approach ensured that the tissues could be made without adversely affecting the mechanical properties of the silicones. The novel use of a sacrificial gelatin core, in particular, was a key design feature of the moulding process, facilitating the thin, continuous accurately formed skin simulant shape. Such a process could theoretically be effectively applied in other areas of future, more complex synthetic human surrogate models for example to generate vascular components.

Although, these techniques were used to manufacture a simplified VHP anatomical dataset with PDMS simulants, the sequential, systematic nature of the process could be effectively applied to any given geometry in a fleshy region of the body surrounding bone with any combination of soft tissue elastomers. The resultant synthetic surrogate illustrates the potential for the construction techniques and materials to be effectively combined.

\section{Further surrogate improvements}

The otherwise successfully moulded full-sized thigh segment did exhibit an unanticipated flaw when released from the mould and left to stand. In medial and posterior regions (Figure 10(c), 10(d)) a crinkling effect developed on the surrogate surface. The location of this problem can be attributed to the increased proportions of the adipose simulant in these regions, which is particularly soft. Several potential reasons for why this may have occurred have been considered:

- Uneven cure rate through volume (e.g. heat source proximity),

- Loss of adipose simulant,

- Adipose simulant shrink back,

- Lack of interstitial fluid pressure, and/or

- Gravitational sagging.

Potential issues relating to the uneven curing rates and loss of adipose simulant in moulding can be easily eliminated through knowledge of the reliability of the environmental chamber used (Alpha 190H, Design Environmental Ltd.) and absence of any overspill from the mould. Similarly, this effect could also have been achieved through an under-fill or a void in the adipose simulant resulting in free space for the material to flow. However, the tissue structure dimensions were physically measured on both superior and distal surfaces and matched the desired dimensions from the CAD model. There could have also been a shrink back effect occurring whereby the skin was compressing the adipose simulant. However, there are no apparent environmental, chemical or physical reasons (e.g. heat shrinking) as to why this may occur.

The more likely reasons for these effects could be explained through examination of real human mechanical behaviour. All humans consist of approximately 16\% interstitial fluid (by mass) which causes a hydrostatic pressure whereby tissues embody an equilibrium tonicity. This pressurisation is clearly absent in the currently proposed surrogate. Another contributory factor is adipose sagging under the influence of gravity caused by a lack of axial tension in the surrogate. The relatively low stiffness of the cured simulant, particularly at quasi-static strain rates, means that it exhibits some 
flow-type behaviour, which could result in deformation under static loads. The discrete, shortened nature of the surrogate thigh could also contribute to this wrinkling effect. The human body consists of a series of continuous interconnected tissue structures with the skin in a state of biaxial tension stretching the local target region. In a discontinuous surrogate body segment, the soft tissues are free to move through the open ends.

\section{Geometric dataset and anatomical simplifications}

The VHP dataset used in this study was acquired from a 38 year old male, which is not representative of an athletic population. The soft tissue thicknesses, in particular, adipose could be expected to be significantly reduced in an athletic demographic, even when considering someone with similar anthropometrics. The VHP geometries were also interpolated from sampled data. Therefore, despite providing a more biofidelic representation of human geometries from previous sports impact surrogates, the model still embodies approximations which could be improved upon. As a first attempt of full-scale synthetic moulding, some simplifications were also made (e.g. increased skin thickness) to ensure that the structures could be moulded.

In most cases, it is desirable to develop a generalised surrogate broadly representative of the target demographic. This approach enables a single, cost-effective surrogate for repeat impact testing of PPE. Therefore future surrogates should focus on better matching the 3D tissue morphologies representative of the intended sports population. However, in spite of the shortcomings of the VHP dataset, the VHP has provided an accessible and practically useful dataset from which to begin to model complex human structures. The presence of complex morphologies and extreme tissue dimensions has demonstrated the potential of the moulding techniques used, possibly beyond what could have been shown in athletic geometries. The adipose wrinkling effects experienced are also likely to be exaggerated by the sedentary VHP male dataset from which the surrogate was moulded.

\section{Applications}

The materials used in human surrogates are the primary components significantly affecting their response to impact. The PDMS simulant materials used have been shown to be more representative of their target organic tissues than previously used single material simulants both individually in standard mechanical specimen tests and combined in finite element (FE) model impact simulations (Payne et al. 2015a, 2015b). Given this, the successful fabrication of these materials in anatomically biofidelic geometries should yield a surrogate representative impact surrogate. This should, in turn, provide a more accurate test structure for the evaluation of PPE.

To most effectively determine the biofidelity of the surrogate it would be necessary to perform impact tests on a subset of humans with comparable anthropometries, particularly in the thigh region. This could ethically be achieved through conducting a series of low intensity impacts with PPE on both human and surrogate bodies (e.g. Halkon et al., 2014). Parallel FE models could also be used to inform the expected biofidelity of the surrogate based on the same CAD geometries and representative organic tissue material models.

Although there is potential to determine impact response through a combination high speed imaging and accelerometers on the striking objects, future improvements to the surrogate may consider embedded instrumentation to provide more direct feedback mechanisms. These could include the use of elastomeric strain gauges and pressure sensors which could be incorporated without adversely affecting the biofidelity of the soft tissue simulants. 


\section{Conclusions}

This research proves that a multiple soft tissue human thigh surrogate can be successfully manufactured for use in sports PPE research. The tissue structures, geometries and simulant materials used have been outlined and development stages in the construction have been sequentially documented. The surrogate demonstrates illustrates the capability of the construction techniques to produce a more representative human test surrogate for any fleshy region of the body surrounding a bone, which could be used to evaluate the effectiveness of PPE for sports applications concerned with soft tissue injuries. Although experimental testing of the surrogate to determine the level of biofidelity achieved remains a future activity, the already proven materials in combination with more anatomical geometries are promising.

\section{Declaration of conflicting interests}

The authors declare that there is no conflict of interest.

\section{Funding}

This research received no specific grant from any funding agency in the public, commercial or notfor-profit sectors.

\section{References}

1. Ackerman, M.J. 1998. The visible human project. Proceedings of the IEEE; 86 (3), pp. 504-511. DOI: 10.1109/5.662875.

2. Alkhouli, N., Mansfield, J., Green, E., Bell, J., Knight, B., Liversedge, N., Tham, J.C., Welbourn, R., Shore, A.C., Kos, K. and Winlove, C.P. 2013. The mechanical properties of human adipose tissues and their relationships to the structure and composition of the extracellular matrix. Am J Physiol Endocrinol Metab.; 305 (12), pp. E1427-35. DOI: 10.1152/ajpendo.00111.2013.

3. Ankrah, S. and Mills, N. 2003. Performance of football shin guards for direct stud impacts. Sports Engineering; 6 (4), pp. 207-219. DOI: 10.1007/BF02844024.

4. Backaitis, S.H. and Mertz, H.J. 1993. Hybrid III: The first human-like crash test dummy. 1st ed. Society of Automotive Engineers: Warrendale, PA.

5. BS 6183-3:2000. 2000. Protective equipment for cricketers - Part 3: Leg protectors for batsmen, wicketkeepers and fielders, and thigh, arm and chest protectors for batsmen. United Kingdom: British Standards Institute.

6. BS:EN 13546:2002. 2002. Protective clothing - Hand, arm, chest. Abdomen, leg, foot and genital protectors for field hockey goal keepers and shin protectors for field players - Requirements and test methods. United Kingdom: British Standards Institute.

7. Chase, M. 1973. Injection training aid. United States. US3722108 A.

8. Chong, A., Miller, F., Buxton, M. and Friis, E.A. 2007. Fracture toughness and fatigue crack propagation rate of short fiber reinforced epoxy composites for analogue cortical bone. Journal of Biomechanical Engineering; 129 (4), pp. 487-493. DOI: 10.1115/1.2746369.

9. Comley, K. and Fleck, N. 2012. The compressive response of porcine adipose tissue from low to high strain rate. International Journal of Impact Engineering; 46 , pp. 1-10. DOI: 10.1016/j.jimpeng.2011.12.009.

10. Conforti, M. 2013. The Treatment of Muscle Hematomas. In: G.N. Bisciotti and C. Eirale eds., Muscle Injuries in Sports Medicine, InTech, pp. 203-220.

11. Cronin, D.S., Williams, K. and Salisbury, C. 2011. Physical surrogate leg to evaluate blast mine injury. Military Medicine; 176 (12), pp. 1408-1416.

12. Crown, E.M., Ackerman, M.Y., Dale, J.D. and Tan, Y. 1998. Design and Evaluation of Thermal Protective Flightsuits. Part II: Instrumented Mannequin Evaluation. Clothing and Textiles Research Journal; 16 (2), pp. 79-87. DOI: 10.1177/0887302X9801600203. 
13. Davison, M., Jones, J. and Allan, T. 2013. Hip Region Soft Tissue Whitepaper. Locus Research \& Delloch Ltd.: Tauranga, New Zealand.

14. Edwards, C. and Marks, R. 1995. Evaluation of biomechanical properties of human skin. Clinics in Dermatology; 13 (4), pp. 375-380. DOI: 10.1016/0738-081X(95)00078-T.

15. Gardner, M.P., Chong, A.C.M., Pollock, A.G. and Wooley, P.H. 2010. Mechanical evaluation of largesize fourth-generation composite femur and tibia models. Annals of Biomedical Engineering; 38 (3), pp. 613-620. DOI: 10.1007/s10439-009-9887-7.

16. Geerligs, M., Peters, G.W., Ackermans, P.A., Oomens, C.W. and Baaijens, F.P. 2008. Linear viscoelastic behavior of subcutaneous adipose tissue. Biorheology; 45 (6), pp. 677-688. DOI: 10.3233/BIR-2008-0517.

17. Goldsmith, L.A. 1990. My organ is bigger than your organ. Archives of Dermatology; 126 (3), pp. 301302. DOI: 10.1001/archderm.1990.01670270033005.

18. Gray, H. 2000. Anatomy of the human body. 20th ed. Lea \& Febiger: Philadelphia.

19. Halkon, B.J., Mitchell, S.R., Payne, T. and Carbo, J. 2014. Biomechanical measurements of human impacts in basketball. Procedia Engineering; 72 , pp. 214-219. DOI: 10.1016/j.proeng.2014.06.038.

20. Hrysomallis, C. 2009. Surrogate thigh model for assessing impact force attenuation of protective pads. Journal of Science and Medicine in Sport; 12 (1), pp. 35-41. DOI: 10.1016/j.jsams.2007.07.013.

21. Miller-Young, J.E., Duncan, N.A. and Baroud, G. 2002. Material properties of the human calcaneal fat pad in compression: experiment and theory. Journal of Biomechanics; 35 (12), pp. 1523-1531. DOI: 10.1016/S0021-9290(02)00090-8.

22. Muggenthaler, H., von Merten, K., Peldschus, S., Holley, S., Adamec, J., Praxl, N. and Graw, M. 2008. Experimental tests for the validation of active numerical human models. Forensic Science International; 177 (2), pp. 184-191. DOI: 10.1016/j.forsciint.2007.12.005.

23. Pailler-Mattei, C., Bec, S. and Zahouani, H. 2008. In vivo measurements of the elastic mechanical properties of human skin by indentation tests. Medical Engineering \& Physics; 30 (5), pp. 599-606. DOI: 10.1016/j.medengphy.2007.06.011.

24. Payne, T., Mitchell, S. and Bibb, R. 2013. Design of human surrogates for the study of biomechanical injury: a review. Critical Reviews ${ }^{\mathrm{TM}}$ in Biomedical Engineering; 41 (1), pp. 51-89. DOI: 10.1615/CritRevBiomedEng.2013006847.

25. Payne, T., Mitchell, S., Bibb, R. and Waters, M. 2015a. Development of novel synthetic muscle tissues for sports impact surrogates. Journal of the Mechanical Behavior of Biomedical Materials; 41 , pp. 357-374. DOI: 10.1016/j.jmbbm.2014.08.011.

26. Payne, T., Mitchell, S., Bibb, R. and Waters, M. 2015b. The evaluation of new multi-material human soft tissue simulants for sports impact surrogates. Journal of the Mechanical Behavior of Biomedical Materials; 41 , pp. 336-356. DOI: 10.1016/j.jmbbm.2014.09.018.

27. Robbins, S.E., Gouw, G.J. and Hanna, A.M. 1989. Running-related injury prevention through innate impact-moderating behavior. Medicine and Science in Sports and Exercise; 21 (2), pp. 130-139.

28. Roberts, J., Merkle, A., Biermann, P., Ward, E., Carkhuff, B., Cain, R. and O’Connor, J. 2007. Computational and experimental models of the human torso for non-penetrating ballistic impact. Journal of Biomechanics; 40 (1), pp. 125-136. DOI: 10.1016/j.jbiomech.2005.11.003.

29. Salem, R.O., Laposata, M., Rajendram, R., Cluette-Brown, J.E. and Preedy, V.R. 2006. The total body mass of fatty acid ethyl esters in skeletal muscles following ethanol exposure greatly exceeds that found in the liver and the heart. Alcohol and Alcoholism (Oxford, Oxfordshire); 41 (6), pp. 598-603. DOI: 10.1093/alcalc/agl069.

30. Saraf, H., Ramesh, K., Lennon, A., Merkle, A. and Roberts, J. 2007. Mechanical properties of soft human tissues under dynamic loading. Journal of Biomechanics; 40 (9), pp. 1960-1967. DOI: 10.1016/j.jbiomech.2006.09.021.

31. Sims, A., Stait-Gardner, T., Fong, L., Morley, J., Price, W., Hoffman, M., Simmons, A. and Schindhelm, K. 2010. Elastic and viscoelastic properties of porcine subdermal fat using MRI and inverse FEA. Biomechanics and Modeling in Mechanobiology; 9 (6), pp. 703-711. DOI: 10.1007/s10237-010-0207-9.

32. Spitzer, V., Ackerman, M.J., Scherzinger, A.L. and Whitlock, D. 1996. The visible human male: a technical report. Journal of the American Medical Informatics Association : JAMIA; 3 (2), pp. 118-130. DOI: 10.1136/jamia.1996.96236280. 
33. Velani, N., Wilson, O., Halkon, B.J. and Harland, A.R. 2012. Measuring the risk of sustaining injury in sport a novel approach to aid the re-design of personal protective equipment. Applied Ergonomics; 43 (5), pp. 883-890. DOI: 10.1016/j.apergo.2011.12.010.

34. Viano, D.C., King, A.I., Melvin, J.W. and Weber, K. 1989. Injury biomechanics research: an essential element in the prevention of trauma. Journal of Biomechanics; 22 (5), pp. 403-417. DOI: 10.1016/0021-9290(89)90201-7.

35. Wang, Z., Deurenberg, P., Wang, W. and Heymsfield, S. 1997. Proportion of adipose tissue-free body mass as skeletal muscle: Magnitude and constancy in men. American Journal of Human Biology; 9 (4), pp. 487-492. DOI: 0.1002/(SICI)1520-6300(1997)9:4<487::AID-AJHB8>3.0.CO;2-T. 